\title{
Heavy ion collider facility NICA at JINR (Dubna): status and development
}

\section{Grigory Trubnikov*, Nikolay Agapov, Alexander Kovalenko, Vladimir Kekelidze, Victor Matveev, Igor Meshkov, Richard Lednicky, Alexander Sorin}

JINR, Joliot Curie, 6, Dubna, Russia, 141980, E-mail: trubnikov@jinr.ru

The project of Nuclotron-based Ion Collider fAcility NICA/MPD (MultiPurpose Detector) under development at JINR (Dubna) is presented. The general goals of the project are providing of colliding beams for experimental studies of both hot and dense strongly interacting baryonic matter and spin physics (in collisions of polarized protons and deuterons). The first program requires providing of heavy ion collisions in the energy range of $\sqrt{ }_{S_{N}}=4 \div 11 \mathrm{GeV}$ at average luminosity of $L=1 \cdot 10^{27} \mathrm{~cm}^{-2} \cdot \mathrm{s}^{-1}$ for ${ }^{197} \mathrm{Au}^{79+}$ nuclei. The polarized beams mode is proposed to be used in energy range of $\sqrt{ }_{s_{N N}}=12 \div 27 \mathrm{GeV}$ (protons) at luminosity of $L \geq 1 \cdot 10^{30} \mathrm{~cm}^{-2} \cdot \mathrm{s}^{-1}$. The key issue of the project is application of cooling methods - stochastic and electron ones. The report contains description of the facility scheme and characteristics in heavy ion operation mode, status and plans of the project development.

36th International Conference of High Energy Physics (ICHEP2012)

July 4-11,

Melbourne, Australia

Speaker 


\section{NUCLOTRON-M \& NICA PROJECT}

The Nuclotron-based Ion Collider fAcility (NICA) [1] is a new accelerator complex (Fig. 1) being constructed at JINR. It is aimed to provide collider experiments with $^{*}$

- heavy ions ${ }^{197} \mathrm{Au}^{79+}$ at $\sqrt{ }_{S_{N N}}=4 \div 11 \mathrm{GeV}(1 \div 4.5 \mathrm{GeV} / \mathrm{u}$ ion kinetic energy) at average luminosity of $1 \cdot 10^{27} \mathrm{~cm}^{-2} \cdot \mathrm{s}^{-1}$ (at $\sqrt{ }_{S_{N N}}=9 \mathrm{GeV}$ );

- light-heavy ions colliding beams of the same energy range and luminosity;

- polarized beams of protons $\sqrt{S}_{s}=12 \div 27 \mathrm{GeV}(5 \div 12.6 \mathrm{GeV}$ kinetic energy) and deuterons ${\sqrt{S_{N N}}}=4 \div 13.8 \mathrm{GeV}(2 \div 5.9 \mathrm{GeV} / \mathrm{u}$ ion kinetic energy) at average luminosity $\geq(1 \div 10) \cdot 10^{30} \mathrm{~cm}^{-2} \cdot \mathrm{s}^{-1}$.

The proposed facility consists of the following elements (Fig. 1):

- “Old” injector (pos. 1): set of light ion sources including source of polarized protons and deuterons and Alvarez-type linac LU-20*);

- "New" injector (under construction): ESIS-type ion source that provides ${ }^{197} \mathrm{Au}^{31+}$ ions of the intensity of $2 \cdot 10^{9}$ ions per pulse of about $9 \mu$ s duration at repetition rate up to $50 \mathrm{~Hz}$ and linear accelerator consisting of RFQ and RFQ Drift Tube Linac (RFQ DTL) sections. The linac accelerates the ions at $A / q \leq 6$ up to the energy of $3 \mathrm{MeV} / \mathrm{u}$ at efficiency not less than $80 \%$.

- Booster-synchrotron housed inside Synchrophasotron yoke (pos. 2). The Booster has superconducting (SC) magnetic system that provides maximum magnetic rigidity of $25 \mathrm{~T} \cdot \mathrm{m}$ at the ring circumference of $211 \mathrm{~m}$. It is equipped with electron cooling system that allows to provide cooling of the ion beam in the energy range from injection energy up to $100 \mathrm{MeV} / \mathrm{u}$. The maximum energy of ${ }^{197} \mathrm{Au}^{31+}$ ions accelerated in the Booster is of $600 \mathrm{MeV} / \mathrm{u}$. Stripping foil placed in the transfer line from the Booster to the Nuclotron allows to provide the stripping efficiency at the maximum Booster energy not less than $80 \%$.

- Nuclotron - SC proton synchrotron (pos. 3) has maximum magnetic rigidity of $45 \mathrm{~T} \cdot \mathrm{m}$ and the circumference of $251.52 \mathrm{~m}$ provides the acceleration of completely stripped ${ }^{197} \mathrm{Au}^{79+}$ ions up to the experiment energy in energy range of $1 \div 4.5 \mathrm{GeV} / \mathrm{u}$ and protons up to maximum energy of $12.6 \mathrm{GeV}$.

- Transfer line (pos. 6) transports the particles from Nuclotron to Collider rings.

- Two SC collider rings (pos. 8) of racetrack shape have maximum magnetic rigidity of $45 \mathrm{~T} \cdot \mathrm{m}$ and the circumference of about $400 \mathrm{~m}$. The maximum field of SC dipole magnets is $1.8 \mathrm{~T}$. For luminosity preservation an electron and stochastic cooling systems will be constructed.

- Two detectors - MultiPurpose Detector (MPD, pos. 9) and Spin Physics Detector $(S P D$, pos. 10) are located in opposite straight sections of the racetrack rings.

- Two transfer lines transport particle beams extracted from Booster and Nuclotron to the new research area, where fixed target experiments both basic and applied character will be placed.

The NICA parameters (Table below) allow us to reach the goals of the project formulated above.

One of NICA accelerators - Nuclotron is used presently for fixed target experiments on extracted beams (Fig. 1, pos. 5). 
This program is planned to be developed further and will be complementary to that one to be performed at Collider in heavy ions beam mode operation. The program includes experimental studies on relativistic nuclear physics, spin physics in few body nuclear systems (with polarized deuterons) and physics of flavours. At the same time, the Nuclotron beams are used for research in radiobiology and applied research.

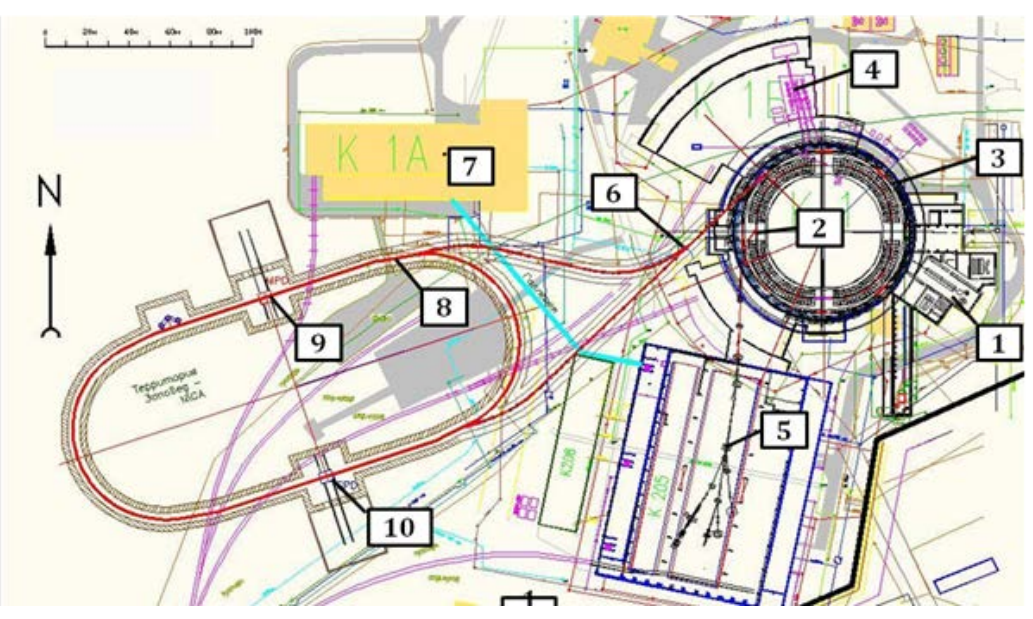

Figure 1: Scheme of NICA facility: 1 - light and polarized ion sources and "old" Alvarez-type linac, ESIS source and new RFQ linac; 2 - Booster inside Synchrophasotron yoke, 3 -

Nuclotron; 4 -cryogenic plant; 5 -Nuclotron beam lines and fixed target experiments; 6 beam transfer lines; 7 - main power converters, 8 - Collider; 9 - Mixed Phase Detector; 10 Spin Physics Detector.

Table of parameters of NICA accelerators

\begin{tabular}{|c|c|c|c|c|}
\hline \multirow[b]{2}{*}{ Acceleration } & \multirow{2}{*}{$\begin{array}{l}\text { Booster } \\
\text { project }\end{array}$} & \multicolumn{2}{|c|}{ Nuclotron } & \multirow{2}{*}{$\begin{array}{l}\text { Collider } \\
\text { project }\end{array}$} \\
\hline & & Project & $\begin{array}{l}\text { Status } \\
\text { (Apr'11) }\end{array}$ & \\
\hline Circumference, m & 211.2 & \multicolumn{2}{|l|}{251.5} & 503.0 \\
\hline Max. magn. field, $\mathrm{T}$ & 2.0 & 2.0 & 2.0 & 1.8 \\
\hline Magn. rigidity, $\mathrm{T} \cdot \mathrm{m}$ & 25.0 & 45 & 39.5 & 45 \\
\hline Cycle duration, s & 4.0 & 4.02 & 5.0 & $\geq 1000$ \\
\hline B-field ramp, T/s & 1.0 & 1.0 & 1.0 & $<0.1$ \\
\hline $\begin{array}{l}\text { Accelerated/stored } \\
\text { particles }\end{array}$ & $\mathrm{p}^{-197} \mathrm{Au}^{79+}$ & $\mathrm{p} \uparrow, \mathrm{d} \uparrow$ & $\mathrm{p}-\mathrm{Xe}, \mathrm{d} \uparrow$ & $\begin{array}{l}\mathrm{p} \div{ }^{197} \mathrm{Au}^{79+}, \mathrm{p} \uparrow, \\
\end{array}$ \\
\hline \multicolumn{5}{|c|}{ Maximum energy, GeV/u } \\
\hline Protons & - & 12.6 & - & 12.6 \\
\hline Deuterons & - & 5.87 & 5.1 & 5.87 \\
\hline Ions, GeV/u & ${ }^{197} \mathrm{Au}^{31+} 0.4$ & ${ }^{197} \mathrm{Au}^{79+} 4.5$ & ${ }^{54} \mathrm{Xe}^{24+} 1.0$ & ${ }^{197} \mathrm{Au}^{79+} 4.5$ \\
\hline \multicolumn{5}{|c|}{ Intensity, ion number per cycle (bunch) } \\
\hline Protons & $1 \cdot 10^{11}$ & $1 \cdot 10^{11}$ & $1 \cdot 10^{11}$ & $1 \cdot 10^{11}$ \\
\hline Deuterons & $1 \cdot 10^{10}$ & $1 \cdot 10^{10}$ & $1 \cdot 10^{10}$ & $1 \cdot 10^{10}$ \\
\hline${ }^{197} \mathrm{Au}^{79+}$ & $2 \cdot 10^{9}$ & $2 \cdot 10^{9}$ & $1 \cdot 10^{6}\left({ }^{54} \mathrm{Xe}^{24+}\right)$ & $1 \cdot 10^{9}$ \\
\hline
\end{tabular}

\section{COLLIDER LUMINOSITY}

The collider design has to provide the project luminosity and its maintenance during a long time necessary for an experiment performance. That requires, correspondingly: 
1) formation of ion beams of high intensity and sufficiently low emittance,

2) ion beam life time.

Beam intensity is limited, in principle, by beam space charge effects, which can be estimated by so called "tune shift criteria". The first one, and most strong of them usually, is so called betatron oscillation tune shift (or "The Laslett tune shift"):

$$
\Delta Q=\frac{Z^{2}}{A} \cdot \frac{r_{p} N_{i}}{\beta^{2} \gamma^{3} 4 \pi \varepsilon_{\text {geom }}} \cdot k_{\text {bunch }}, k_{\text {bunch }}=\frac{C_{\text {Ring }}}{\sqrt{2 \pi} \cdot \sigma_{s}} .
$$

Here $Z$ and $A$ are ion charge and mass number, $r_{p}$ is proton classic radius, $N_{i}$ is ion number per bunch in the bunched ion beam, $\beta, \gamma$ are the ion Lorentz factors, $k_{\text {bunch }}$ is bunch factor, $C_{R i n g}$ is the Collider ring circumference, $\sigma_{s}$ is bunch length ( $\sigma$-value for Gaussian beam), $\varepsilon_{\text {geom }}$ is the ion bunch "geometrical" transverse emittance (do distinguish with "normalized" one $\varepsilon_{\text {norm }}$ used below). The second criterion is so called beam-beam parameter that describes ion betatron tune shift related to scattering of ion on the electromagnetic field of encountering ion bunch:

$$
\xi=\frac{Z^{2}}{A} \cdot \frac{r_{p} N_{i}\left(1+\beta^{2}\right)}{4 \pi \beta^{2} \gamma \varepsilon_{\text {geom }}}
$$

For practical estimates one can use the numerical criterion for beam stability as follows:

$$
\Delta Q_{\text {total }} \equiv \Delta Q+n_{\xi} \xi \leq 0.05 \text {. }
$$

$n_{\xi}$ is number of interaction points.

One of instabilities and major problems of the NICA collider is suppression of intrabeam scattering (IBS) in intense ion bunches. The last one defines mainly the beam life time. For this purpose we have proposed to use both electron cooling [2] and stochastic cooling [3] methods. In the first case we assume achievement of an equilibrium between cooling and space charge forces when space charge tune shift $\Delta Q_{\text {total }}$ reaches a resonant value (e. g., 0.05). We call it space charge dominated regime (SCD regime). Then using Formulae (1), (2) and well known expression for luminosity of round colliding beams one can derive simple relations between parameters:

$$
L \propto \Delta Q_{\text {total }}^{2} \cdot \varepsilon_{\text {geom }} \cdot f_{L}\left(E_{\text {ion }}\right) \cdot f_{H G}, N_{i} \propto \Delta Q_{\text {total }} \cdot \varepsilon_{\text {geom }} \cdot f_{N}\left(E_{\text {ion }}\right),
$$

where $E_{i}$ is ion energy, $f_{L}, f_{n}$ are the functions describing energy dependence of parameters, $f_{H G}$ is hour-glass effect function. We see that maximum luminosity is achieved if beam emittance $\varepsilon_{\text {geom }}$ has maximum, i. e. coincides with the ring acceptance. At some circumstances the luminosity can be limited by "not the beam reasons (e. q., detector performance). Then one can optimise the SCD regime decreasing equilibrium emittance and $N_{i}$ (Fig. 2). Such an optimisation can be done with variation of $N_{i}$ number. In the case of limited luminosity one can also avoid SCD 
regime decreasing ion number and allowing, by weakening cooling force, the beam emittance keeping $\Delta Q_{\text {total }}$ below resonant value. We call it IBS Dominated regime (IBS $\mathrm{DR}$ ) when equilibrium state is provided with equality IBS and cooling rates:

$$
\mathrm{R}_{\mathrm{IBS}}=\mathrm{R}_{\mathrm{cool}} \text {. }
$$
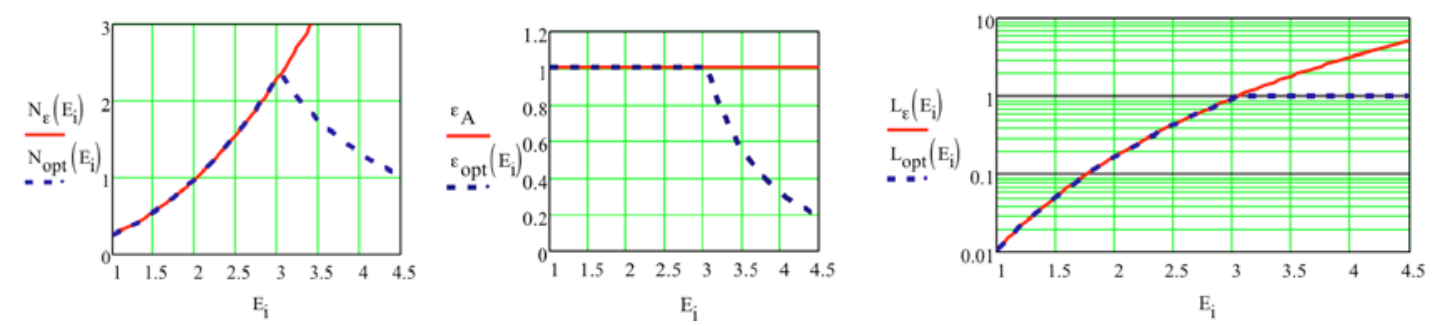

Figure 2: Space charge dominated regime; ion number per bunch (a), beam emittance (b) and luminosity (c) versus ion energy in two cases: full acceptance if filled with ions (red solid curves) and luminosity is limited (blue dash curve); the ring acceptance $=40 \pi \cdot \mathrm{mm} \cdot \mathrm{mrad}$, parameter units:

$$
\left[N_{i}\right]=10^{9},[\varepsilon]=\pi \cdot \mathrm{mm} \cdot \mathrm{mrad},[\mathrm{L}]=10^{27} \mathrm{~cm}^{-2} \cdot \mathrm{s}^{-1}
$$

Then, at fixed luminosity, similar by to Formulae (4) one can write

$$
L=\text { const, } N_{i} \propto \sqrt{L \cdot \varepsilon_{\text {geom }}} \cdot \varphi_{L}\left(E_{i}\right), \Delta Q_{\text {total }} \propto \sqrt{\frac{L}{\varepsilon_{\text {geom }}}} \cdot \psi_{L}\left(E_{i}\right)<\Delta Q_{\text {max }} \cdot
$$

As we see, minimum $\Delta Q_{\text {total }}$ corresponds to maximum emittance, i. e. full acceptance filling with ions. Simultaneously, it gives us maximum $\tau_{I B S}$ at relatively increased ion number (Fig. 3). One should mention that at IBS DR ion number dependence of energy is rather weak - proportional to $\left(N_{\text {ion }} / C\right)^{-1 / 2}$.

For NICA parameters, as it follows from Fig. 3, IBS DR regime can be used at $E_{i}>3 \mathrm{GeV} / \mathrm{u}$ where $\Delta Q<0.05$. At the same energy range we plan to use stochastic cooling. At lower energy electron cooling application is preferable (if not to say more realizable) [3, 2]. However, then another problem appears: ion recombination with cooling electrons. This effect can be significantly diminished by increase of cooling electrons temperature [4].

The described approach (SC and IBS dominated regimes) can be developed even further. One can, for instance, increase luminosity in low energy range (below $3 \mathrm{GeV} / \mathrm{u}$ ) by enlarging minimum beta-functions in IP area. That will be followed by decrease of beta-functions in the lenses of final focus and lead correspondingly to increase of the ring acceptance. Those steps are planned for future development. 


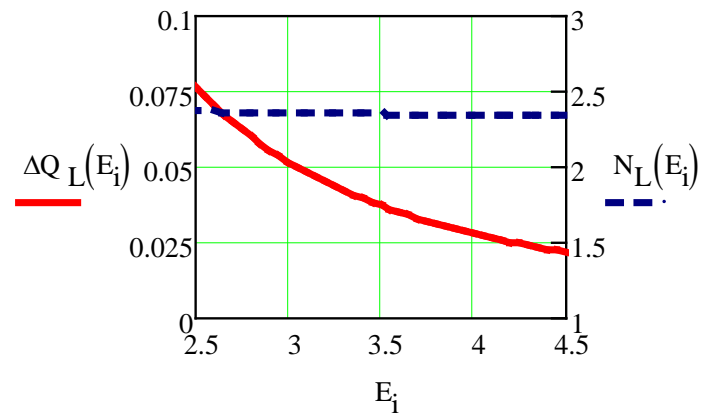

Figure 3: IBS dominated regime; beam tune shift $\Delta Q_{\text {total }}$ (red solid curve) and ion number per bunch $N_{i}$ (blue dash curve) at constant luminosity $L=1 \cdot 10^{27} \mathrm{~cm}^{-2} \cdot \mathrm{s}^{-1}$ and beam emittance of $1.0 \pi \cdot \mathrm{mm} \cdot \mathrm{mrad}$;

$$
\left[N_{\mathrm{i}}\right]=10^{9} \text {. }
$$

\section{NICA CRYOGENIC SYSTEM}

The NICA cryogenics (Fig. 4) will be based on the modernized liquid helium plant that was built in the early 90's for the Nuclotron. The main goals of the modernization consist of increasing of the total refrigerator capacity from $4000 \mathrm{~W}$ to $8000 \mathrm{~W}$ at $4.5 \mathrm{~K}$ and construction a new distribution system of liquid helium. These goals will be achieved by construction of a new $1000 \mathrm{l} / \mathrm{hour}$ helium liquefier, "satellite" refrigerators located near the accelerator rings, and a liquid nitrogen system that will be used for shield refrigerating at $77 \mathrm{~K}$ and at the first stage of cooling down of three accelerator rings with the total length of about $1.5 \mathrm{~km}$ and "cold" mass of 220 tons.

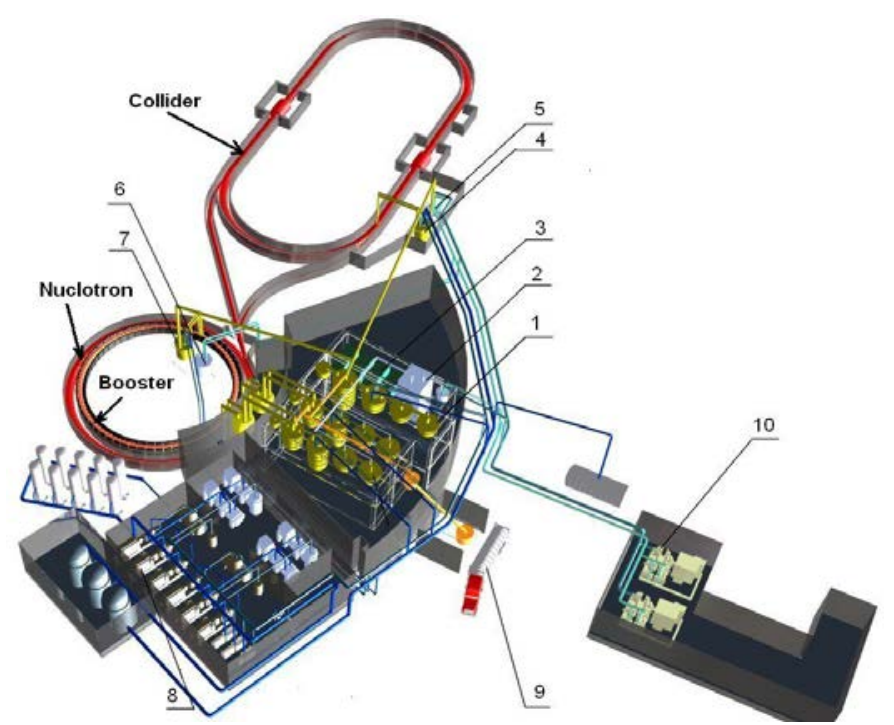

Figure 4. The general view of the NICA cryogenic system. New units for the NICA accelerators: 1 $1000 \mathrm{l} / \mathrm{h}$ Helium liquefier OG-1000; 2 - 1300 kg/h Nitrogen liquefier OA-1,3; 3 - draining and oilpurification units; 4 - satellite refrigerator of the collider; 5 - $500 \mathrm{~kg} / \mathrm{h}$ Nitrogen re-condenser RA-0,5 of the collider; 6 - $500 \mathrm{~kg} / \mathrm{h}$ Nitrogen re-condenser RA-0,5 of the Booster; 7 - satellite refrigerator of the Booster; $8-6600 \mathrm{~N} \cdot \mathrm{m}^{3} / \mathrm{h}$ screw compressors Kaskad-110/30; 9 - liquid Helium tank; 10 - Nitrogen turbo compressors. 


\section{CONCLUSION}

The status main characteristics of NICA project and principle problems related to the NICA collider creation are considered in this report. The NICA project as a whole has passed the phase of concept formulation and is presently under development of the working project, manufacturing and construction of the prototypes.

The project realization plan foresees a staged construction and commissioning of the accelerators that form the facility. The main goal is beginning of the facility commissioning in 2017.

\section{References}

[1] Search for a QCD Mixed Phase at the Nuclotron-based Ion Collider fAcility (NICA White Paper), http://theor.jinr.ru/twiki/pub/NICA/WebHome/WhitePaper_5.03.pdf.

[2] S. Yakovenko, E. Ahmanova, A. Kobets et al., Cooler for Collider NICA, Proc. of COOL'2011, TUPS13.

[3] G. Trubnikov, Application of Cooling Methods in the NICA Project, Proc. of COOL'2011, MOIO07.

[4]A. Philippov, A. Kuznetsov, I. Meshkov, Radioactive Recombination of Bare Nuclei and Ions in Electron Cooling System, Proc. of COOL'2011, THCOB01 